\title{
Implementation of a multicomponent telemonitoring intervention to improve nutritional status of community-dwelling older adults: a process evaluation
}

\author{
Marije N van Doorn-van Atten ${ }^{1, *}$, Lisette CPGM de Groot ${ }^{1}$, Albert Collet Romea ${ }^{2}$, \\ Simon Schwartz ${ }^{2}$, Jeanne HM de Vries $^{1}$ and Annemien Haveman-Nies ${ }^{1,3}$ \\ 'Wageningen University \& Research, Division of Human Nutrition, PO Box 17, 6700 AA Wageningen, The \\ Netherlands: ${ }^{2}$ Meditecnologia, Barcelona, Spain: ${ }^{3}$ Wageningen University \& Research, Strategic Communication \\ Chair, Wageningen, The Netherlands
}

Submitted 8 March 2018: Final revision received 18 July 2018: Accepted 1 August 2018: First published online 3 September 2018

\begin{abstract}
Objective: The present study aimed to conduct a process evaluation of a multicomponent nutritional telemonitoring intervention implemented among Dutch community-dwelling older adults.

Design: A mixed-methods approach was employed, guided by the process evaluation framework of the Medical Research Council and the Unified Theory of Acceptance and Use of Technology. The process indicators reach, dose, fidelity and acceptability were measured at several time points within the 6-month intervention among participants and/or nurses.

Setting: The intervention was implemented in the context of two care organisations in the Netherlands.

Subjects: In total, ninety-seven participants (average age 78 years) participated in the intervention and eight nurses were involved in implementation.

Results: About $80 \%$ of participants completed the intervention. Dropouts were significantly older, had worse cognitive and physical functioning, and were more care-dependent. The intervention was largely implemented as intended and received well by participants (satisfaction score $4 \cdot 1$, scale $1-5$ ), but less well by nurses (satisfaction score 3.5, scale 1-5). Participants adhered better to weight telemonitoring than to telemonitoring by means of questionnaires, for which half the participants needed help. Intention to use the intervention was predicted by performance expectancy $(\beta=0.40 ; 95 \%$ CI $0.13,0.67)$ and social influence $(\beta=0 \cdot 17 ; 95 \%$ CI $0 \cdot 00,0 \cdot 34)$. No association between process indicators and intervention outcomes was found.

Conclusions: This process evaluation showed that nutritional telemonitoring among older adults is feasible and accepted by older adults, but nurses' satisfaction should be improved. The study provided relevant insights for future development and implementation of eHealth interventions among older adults.
\end{abstract}

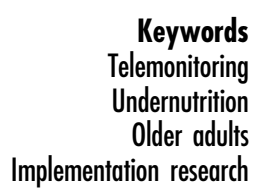

Keywords Undernutrition Older adults Implementation research
Undernutrition impedes healthy ageing as it has been associated with increased morbidity and mortality ${ }^{(1)}$. It is estimated that $5 \cdot 8 \%$ of community-dwelling older adults are undernourished and another $31.8 \%$ are at risk of undernutrition $^{(2)}$. Among home care clients the estimated prevalence of undernutrition is $35 \%{ }^{(3)}$. Nevertheless, it is stated that undernutrition is "under-recognized and undertreated $^{,(4)}$. Nutrition education and nutritional monitoring may improve awareness among older adults and healthcare professionals and may lead to timely detection and prevention $^{(1)}$.
In previous research the effectiveness of the PhysioDom Home Dietary Intake Monitoring (HDIM) intervention was studied $^{(5)}$. This intervention consisted of telemonitoring, nutrition education and follow-up by a nurse, and was implemented in a health-care setting among Dutch community-dwelling older adults. The intervention improved nutritional status in participants at risk of undernutrition and improved diet quality. No effects on physical functioning and quality of life were found ${ }^{(5)}$.

Besides effect evaluation of such a complex, multicomponent intervention, process evaluation of PhysioDom 
HDIM is indispensable. First, insights from a process evaluation guide implementation quality and ensure that the intervention is carried out as intended ${ }^{(6)}$. Second, research to the context, implementation and mechanisms of impact of PhysioDom HDIM is crucial to interpret findings from the effect evaluation and to implement the intervention in another setting ${ }^{(7)}$. Third, policy makers frequently highlight the role of technology in supporting ageing in place and effort is put into developing technology to improve health and self-management of diseases ${ }^{(8)}$. However, several barriers hinder successful implementation and widespread adoption of eHealth among older adults is lacking ${ }^{(9)}$. Research on eHealth adoption by older adults has mostly focused on the pre-implementation stage and often comprised qualitative studies ${ }^{(10)}$. More research is needed to understand what factors contribute to sustained use of eHealth. Therefore, process evaluation of the PhysioDom HDIM intervention could provide insight into what contributes to successful eHealth adoption by older adults. The aims of the present paper were to study how PhysioDom HDIM was delivered and received by participants and nurses, and to study the intervention's mechanisms of impact.

\section{Methods}

\section{Theoretical framework}

A mixed-methods approach was employed guided by the framework of the Medical Research Council ${ }^{(7,11)}$. Based on this framework, we included the process indicators reach, dose, fidelity and acceptability. Acceptability was studied in further detail by using the Unified Theory of Acceptance and Use of Technology (UTAUT). UTAUT is a widely used framework that unifies several technology acceptance models into one, explaining up to $70 \%$ of intention to use technology through four factors: performance expectancy, effort expectancy, social influence and facilitating conditions ${ }^{(11)}$. UTAUT has been widely applied in research also among older adults ${ }^{(11-14)}$ and is helpful in understanding the drivers of acceptance and in designing interventions that will be optimally used $^{(11)}$.

\section{Study design}

The current process evaluation was conducted as part of a 6-month intervention study which followed a parallel arm pre-test/post-test design and took place from April 2016 until June $2017^{(15)}$. We used data from the intervention group only. Measurements were conducted at baseline (T0), 4.5 months after the start of the study (T1) and 6 months after the start of the study (T2). Furthermore, continuous implementation monitoring took place through log data and registration of study procedures by researchers and nurses. The study was registered at ClinicalTrials.gov (identifier NCT03240094; http://bit.ly/2zFTs3P).

\section{Participants}

Participants were recruited from the municipalities of Ermelo, Harderwijk, Nunspeet, Putten and Renkum in the Netherlands. They were invited via advertisements in local newspapers and public spaces, post and letters from the care organisations Zorggroep Noordwest-Veluwe and Opella. Persons could respond when they were 65 years or older and received home care and/or lived in a service flat or sheltered accommodation. Interested persons were visited by a researcher to receive more information about the study, ask questions, sign the informed consent and be screened on the exclusion criteria. Persons were excluded if they were cognitively impaired (Mini Mental State Examination (MMSE) score $<20$ ), had diagnosed cancer, received terminal care, were bedridden or bound to a wheelchair, or were unable to watch television. In total, 107 persons were screened on eligibility for participation in the intervention group, of whom ninety-seven were allocated to the intervention group. During the intervention period, twenty-one participants were lost to followup. This was mainly due to health problems ( $n$ 10) or difficulties with the telemonitoring technology ( $n 5$ ).

\section{Intervention}

The PhysioDom HDIM intervention consisted of three components: nutritional telemonitoring, nutrition education, and follow-up of telemonitoring measurements by a nurse. These components are explained briefly below; a full description can be found elsewhere ${ }^{(15)}$. Participants performed self-measurements of body weight (weekly), steps taken during a day (one week per month) and blood pressure (monthly or bimonthly, only upon indication of a nurse). Participants also filled out questionnaires about nutritional status, appetite and diet quality using the Mini Nutritional Assessment Short-Form (MNA-SF) ${ }^{(16)}$, the Simplified Nutritional Appetite Questionnaire (SNAQ) ${ }^{(17)}$ and the Dutch Healthy Diet Food Frequency Questionnaire (DHD-FFQ) ${ }^{(18)}$, respectively (at T0 and three months later). Participants could do this on a tablet, on their own computer, or via a telephone interview with researchers, and were trained for this during an individual at-home training at T0. A helpdesk was available to support participants if they encountered difficulties. Furthermore, participants received three television messages per week containing general information about nutrition and physical activity. These short text messages ( $<500$ characters) were displayed on a special television channel. Participants also received two letters with tailored information about how to improve compliance with Dutch guidelines for diet and physical activity. Tailoring was based on an individual's DHD-FFQ results: for each guideline, one out of two to five available advices was given, according to the 
score for that specific guideline. Finally, a team of eight nurses and three dietitians assessed the telemonitoring results. Nurses viewed the results on a website and checked the alerts that were activated in case of undernutrition, risk of undernutrition, obesity or new blood pressure measurements. Nurses decided about proper follow-up with the help of decision trees ${ }^{(15)}$. In case of risk of undernutrition, nurses investigated the causes and advised participants on how to improve protein and energy intakes ${ }^{(19)}$. In case of undernutrition and obesity, nurses discussed with participants whether referral to a general practitioner or dietitian was desired. In case of deviating blood pressure measurements, nurses followed regular care pathways. Nurses were trained during four preparatory meetings of $1.5 \mathrm{~h}$ with the researchers. They also attended a workshop from a dietitian to improve knowledge about nutrition in older adults. The researchers held monthly to bimonthly telephone meetings with nurses to address questions and to ensure proper implementation.

\section{Measurements}

\section{Reach}

Reach is defined as 'proportion of the intended priority audience that participates in the intervention, ${ }^{(6)}$. Reach was investigated by keeping a logbook of dropout and by collecting background characteristics of participants. Sex, age, BMI, education level, civil status, living situation, number of diagnoses, cognitive functioning as measured by the $\mathrm{MMSE}^{(20)}$, the presence of dental and/or swallowing problems and type of care were recorded during a screening visit before T0. Other characteristics were measured at T0, including nutritional status, measured by the Mini Nutritional Assessment (MNA) ${ }^{(21)}$, and physical functioning, measured by the Short Physical Performance Battery and the Katz-15 $5^{(22,23)}$.

\section{Fidelity}

Fidelity is defined as 'the extent to which an intervention was implemented as planned' and was assessed by keeping a logbook of study procedures and a paper questionnaire for nurses ${ }^{(6)}$. This questionnaire was filled out half-way during the project and contained questions on how much time the health-care professional spent on the project and how often the health-care professional used the project website.

\section{Dose received}

Dose received is defined as the extent to which participants actively engage with, interact with, are receptive to, and/or use materials or recommended resources ${ }^{,(6)}$. Dose received was measured by log data from the television channel and project website, paper questionnaires for participants and nurses, and registration lists of nurses. With log data the proportion of requested weight and step count measurements that participants actually performed was measured. It should be noted that participants also wrote down their steps on paper, so log data only partially reveal dose received concerning step counts. The questionnaire for participants was filled out at T1 and T2 and contained questions on the frequency of reading television messages and telemonitoring of body weight. The questionnaire for nurses was filled out half-way during the project and included the question 'How long on average did the contact moments take with participants with risk of undernutrition?'

\section{Acceptability}

Acceptability of the intervention was studied by using the UTAUT model $^{(11)}$. UTAUT constructs were measured at T1 and $\mathrm{T} 2$ by paper questionnaires for participants. The questionnaires contained statements that were answered on a five-point Likert scale ranging from 'completely disagree' to 'completely agree'. Performance expectancy is defined as 'the degree to which an individual believes that using the system will help him or her to attain gains in job performance' and was translated into 'gains in health behaviour or health' to fit in the context of the study. Performance expectancy was measured using the following statements: 'The project helps me to be more physically active', 'The project helps me to eat healthier' and 'The project improved my health'. Effort expectancy is defined as 'the degree of ease associated with the use of the system' and was measured using the following statements: 'Working with the television channel is easy' and 'It is easy to weigh myself/use the pedometer/use the remote control/use the tablet/use the sphygmomanometer'. Social influence is defined as 'the degree to which important others believe he or she should use the new system' and was measured with the following statements: 'My partner/ family/friends/others support me in participating in the project' and 'The support of my partner/family/friends/ others is important to me'. Facilitating conditions are defined as 'the degree to which an individual believes that an organisational and technical infrastructure exists to support the use of the system' and was studied by several statements concerning the satisfaction about the helpdesk and the training. Behavioural intent was measured at T2 with one statement: 'I would like to use the intervention more often'. Furthermore, semi-structured interviews with fifteen participants were performed to gain more in-depth insight into acceptability. The interviews took on average 30 min, took place during T2 at the participants' homes and were guided by an interview guide (Table 1). After verbal consent, all interviews were recorded and transcribed verbatim.

Acceptability of nurses was also assessed with help of the UTAUT model. Nurses filled out a paper questionnaire halfway during the project with statements that were answered on a five-point Likert scale ranging from 'completely disagree' to 'completely agree'. The construct performance 
Table 1 Interview guide for interviews with participants $(n$ 15) of the PhysioDom HDIM interventiont in the Netherlands

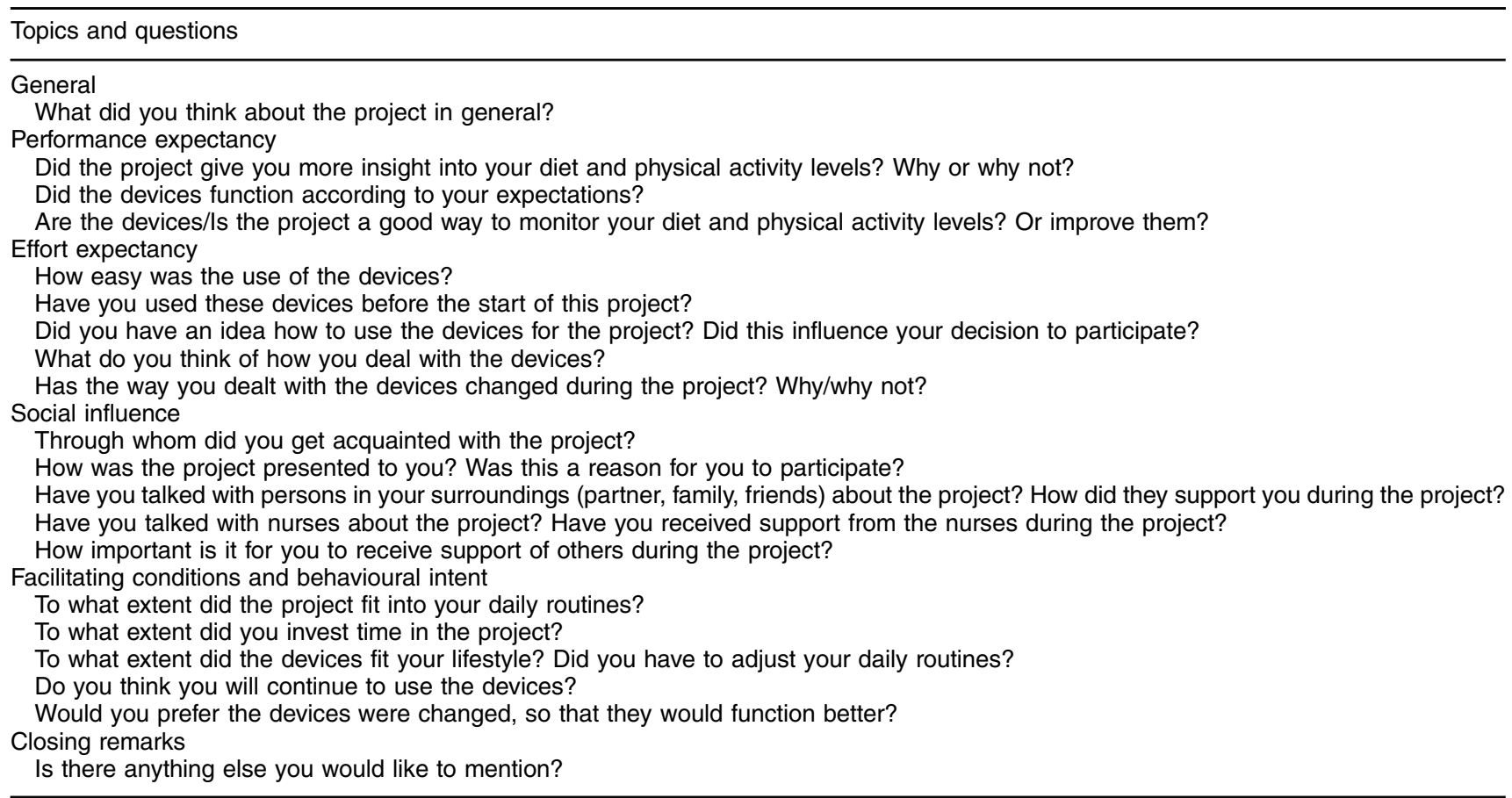

†The PhysioDom Home Dietary Intake Monitoring (HDIM) intervention consisted of telemonitoring, nutrition education and follow-up by a nurse, implemented in a health-care setting among Dutch community-dwelling older adults from April 2016 to June 2017.

expectancy was divided into two sub-constructs, 'gains for job performance' and 'gains for client'. Gains for job performance contained six statements concerning the added value of the intervention for the job performance of the health-care professional, for example: 'Through the intervention I can do my work more efficiently'. Effort expectancy consisted of ten items concerning the ease of use of the project website. Social influence was assessed by two items concerning the support of colleagues. Behavioural intent contained the statement: 'I would like to participate in a continuation of the project'. Furthermore, semi-structured interviews of 20-30 min were held with each of the nurses including acceptability topics (Table 2). After verbal consent, interviews were recorded and transcribed verbatim.

\section{Explaining mechanisms of impact}

To study the mechanisms of impact, the associations of participant characteristics and the process indicator acceptability with intention to use the intervention were examined. Furthermore, the association of the process indicators acceptability and dose received with changes in the outcomes that were significantly affected by the intervention was examined. These were nutritional status and compliance to Dutch dietary guidelines for the intake of fruits, vegetables, dietary fibre and protein, and compliance to guidelines for physical activity ${ }^{(5)}$. Nutritional status was measured using the MNA during a structured
Table 2 Interview guide for interviews with nurses $(n$ 8) implementing the PhysioDom HDIM interventiont in the Netherlands

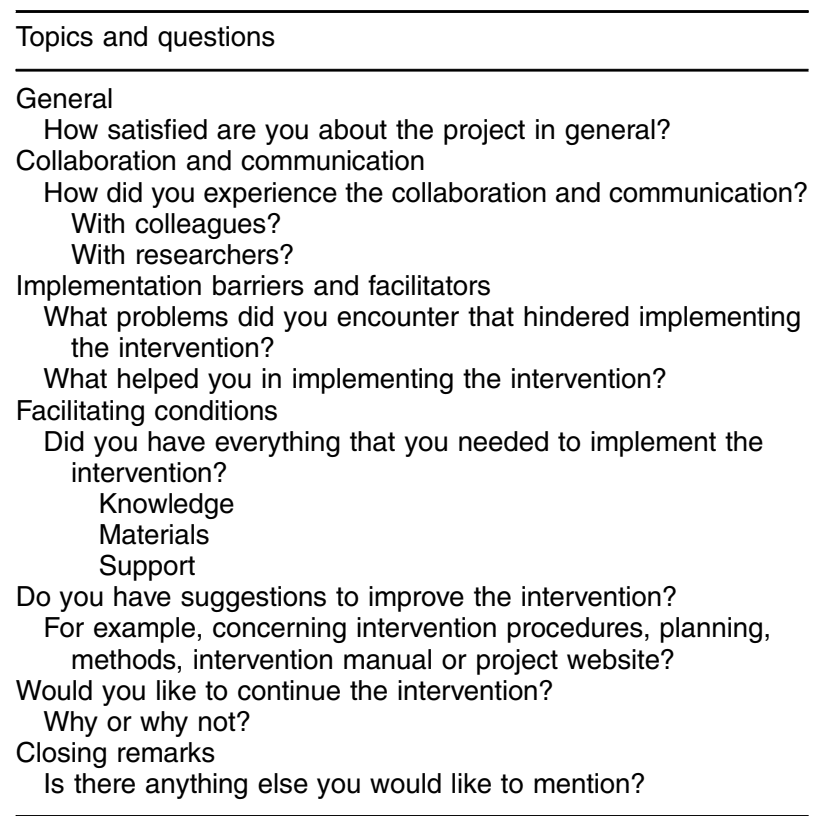

†The PhysioDom Home Dietary Intake Monitoring (HDIM) intervention consisted of telemonitoring, nutrition education and follow-up by a nurse, implemented in a health-care setting among Dutch community-dwelling older adults from April 2016 to June 2017.

interview with participants at $\mathrm{T} 0, \mathrm{~T} 1$ and $\mathrm{T} 2{ }^{(21)}$. Body weight was measured at T0, T1 and T2 by researchers using a weighing scale (brand A\&D, type UC-411PBT-C). 
Participants were asked to take off their shoes and heavy clothes such as a jacket before weighing. Diet quality was assessed using the DHD-FFQ during a structured interview with participants at $\mathrm{T} 0$ and $\mathrm{T} 2{ }^{(18)}$. The DHD-FFQ contains twenty-eight items to evaluate compliance to Dutch dietary guidelines for vegetables, fruits, fish, alcohol, saturated fatty acids, trans-fatty acids, $\mathrm{Na}$ and dietary fibre, and compliance to Dutch guidelines for physical activity ${ }^{(24)}$.

\section{Statistics}

Data were analysed using the statistical software package IBM SPSS Statistics for Windows version 22. Baseline characteristics were analysed using descriptive statistics. Differences in baseline characteristics between completers and dropouts were analysed using independent $t$ tests or $\chi^{2}$ tests. The process indicators dose received and acceptability were analysed using descriptive statistics, showing percentages or means with standard deviations. Cronbach's $\alpha$ was used to investigate whether acceptability items could be combined into the UTAUT constructs. If Cronbach's $\alpha$ was lower than 0.70 , items were presented separately. The association of participant characteristics and acceptability with intention to use the intervention was analysed using a nested linear regression analysis, including participant characteristics in the first block, then adding the four UTAUT constructs blockwise to the model. An $F$ test revealed whether adding these constructs significantly increased the explained variance. Associations of process indicators and changes in health and behavioural outcomes were analysed using linear regression. Qualitative data were analysed using the software Atlas.ti version 7. Interview transcripts were coded deductively using codes from UTAUT constructs. New codes were generated for relevant sections that did not belong to any of the UTAUT constructs. Thematic analysis was used to study factors that influenced acceptance of the intervention. Interviews with the nurses were analysed by grouping relevant sections, phrases, sentences or words into themes.

\section{Results}

\section{Reach}

Table 3 shows the baseline characteristics of the study population. Twenty-one of ninety-seven participants dropped out of the study. They were significantly older,

Table 3 Reach of the PhysioDom HDIM interventiont in terms of baseline characteristics of the total group of participants, participants who completed the study and participants who dropped out

\begin{tabular}{|c|c|c|c|c|c|c|c|}
\hline & \multicolumn{2}{|c|}{ Total $(n 97)$} & \multicolumn{2}{|c|}{ Completers ( $n 76)$} & \multicolumn{2}{|c|}{ Dropouts ( $n 21)$} & \multirow[b]{2}{*}{$P$ value } \\
\hline & Mean & SD & Mean & SD & Mean & SD & \\
\hline Age (years) & $78 \cdot 4$ & $7 \cdot 2$ & $77 \cdot 3$ & $7 \cdot 2$ & $82 \cdot 3$ & $6 \cdot 1$ & $<0.01$ \\
\hline $\mathrm{BMI}\left(\mathrm{kg} / \mathrm{m}^{2}\right)$ & 29.2 & $4 . \overline{5}$ & $29 \cdot 0$ & $4 \cdot \overline{1}$ & 29.6 & 5.9 & 0.66 \\
\hline Number of diagnoses & 1.5 & 1.5 & 1.7 & 1.6 & 1.0 & $1 \cdot 1$ & 0.06 \\
\hline MMSE score $\ddagger$ & 28.6 & 1.5 & $29 \cdot 0$ & 1.2 & $27 \cdot 2$ & 1.9 & $<0.001$ \\
\hline SPPB score & $7 \cdot 2$ & $3 \cdot 1$ & 7.6 & $2 . \overline{9}$ & $5 \cdot \overline{4}$ & 3.2 & $<0.01$ \\
\hline \multirow[t]{2}{*}{ Katz-15 score§ } & $2 \cdot 1$ & $2 \cdot 7$ & 1.9 & 2.6 & $3 \cdot 2$ & $2 \cdot 8$ & 0.07 \\
\hline & \multicolumn{2}{|c|}{$\%$} & \multicolumn{2}{|c|}{$\%$} & \multicolumn{2}{|c|}{$\%$} & \\
\hline Sex (male) & \multirow{2}{*}{\multicolumn{2}{|c|}{$34 \cdot 0$}} & \multicolumn{2}{|c|}{31.6} & \multicolumn{2}{|c|}{$42 \cdot 9$} & 0.33 \\
\hline Education level|| & & & & & & 0.56 \\
\hline Low & \multicolumn{2}{|c|}{$17 \cdot 5$} & \multicolumn{2}{|c|}{$18 \cdot 4$} & \multicolumn{2}{|c|}{$14 \cdot 3$} & \\
\hline Intermediate & \multicolumn{2}{|c|}{$55 \cdot 7$} & \multicolumn{2}{|c|}{$52 \cdot 6$} & \multicolumn{2}{|c|}{$66 \cdot 7$} & \\
\hline High & \multirow{2}{*}{\multicolumn{2}{|c|}{$\begin{array}{l}26.8 \\
55.7\end{array}$}} & \multicolumn{2}{|c|}{28.9} & \multicolumn{2}{|c|}{$19 \cdot 0$} & \\
\hline Living alone & & & \multirow{2}{*}{\multicolumn{2}{|c|}{$59 \cdot 2$}} & \multicolumn{2}{|c|}{$42 \cdot 9$} & 0.22 \\
\hline Desire to lose weight§ & \multicolumn{2}{|c|}{$52 \cdot 7$} & & & \multicolumn{2}{|c|}{$50 \cdot 0$} & 1.0 \\
\hline Currently on a diet§ & \multicolumn{2}{|c|}{9.7} & \multicolumn{2}{|c|}{$\begin{array}{r}53 \cdot 3 \\
9 \cdot 3\end{array}$} & & & 1.0 \\
\hline Nutritional status $\ddagger$ & & & & & & 0.81 \\
\hline Normal nutritional status & \multirow{2}{*}{\multicolumn{2}{|c|}{$\begin{array}{c}79.2 \\
19.8\end{array}$}} & \multicolumn{2}{|c|}{$77 \cdot 6$} & \multicolumn{2}{|c|}{85.0} & \\
\hline At risk of undernutrition & & & \multicolumn{2}{|c|}{$21 \cdot 1$} & & & \\
\hline Undernourished & & & & & & & \\
\hline Type of care (more than on & per part & poss & & & & & \\
\hline Domestic care & & & & & & & 0.55 \\
\hline Personal care & & & & & & & $<0.01$ \\
\hline Nursing care & & & & & & & 0.02 \\
\hline Individual support & & & & & & & 0.52 \\
\hline Informal care & & & & & & & 0.80 \\
\hline
\end{tabular}

MMSE, Mini-Mental State Examination; SPPB, Short Physical Performance Battery.

†The PhysioDom Home Dietary Intake Monitoring (HDIM) intervention consisted of telemonitoring, nutrition education and follow-up by a nurse, implemented in a health-care setting among Dutch community-dwelling older adults from April 2016 to June 2017.

¥One missing value.

§Four missing values.

\|Low education level: primary school or less; intermediate level of education: secondary professional education or vocational school; high education level: higher vocational education, university. 
had a lower cognitive and physical functioning, and were more care-dependent than participants who completed the study. Reasons for dropout were health problems ( $n$ 10), difficulties with the technology ( $n$ 5), inability to install the telemonitoring technology properly due to technical problems ( $n$ 2), health problems of spouse $(n 1)$, dislike of the intervention ( $n$ 1) or reason for dropout was unknown $(n 2)$.

\section{Fidelity}

\section{Telemonitoring measurements}

Adherence to telemonitoring measurements was high for body weight and lower for steps, nutritional status, appetite and diet quality (see 'Dose' subsection below). Half of the participants omitted to perform at least one of the telemonitoring questionnaires, so that researchers had to assist them with filling these out at T1. Furthermore, some participants needed nurses' assistance with telemonitoring measurements, while it was the intention that participants would be able to perform these measurements independently.

\section{Nutrition education}

According to the intervention plan, participants received three television messages per week and two letters with computer-tailored advice about diet quality and physical activity.

\section{Follow-up by the nurse}

Nurses provided follow-up on the telemonitoring alerts according to the intervention plan. It was planned that this would take $0.75 \mathrm{~h} /$ week. However, the project took nurses on average $1.26 \mathrm{~h} /$ week (range $0.5-3.0 \mathrm{~h} /$ week). It was not specified how this time was distributed over the different intervention tasks, but part of this time might have been spent on the additional help that was needed with telemonitoring measurements. Half of the nurses checked the project website less often than once per week as agreed upon with the researchers, mentioning a lack of time as reason for this.

\section{Dose}

Table 4 shows participants' adherence to the telemonitoring measurements. Either with or without help from nurses or researchers, participants performed on average $70 \%$ of the body weight measurements, $37 \%$ of the step count measurements, and $100 \%$ of the measurements of nutritional status, appetite and diet quality. A little under half of the participants indicated to have read the

Table 4 Dose of the PhysioDom HDIM interventiont components received by the intervention group

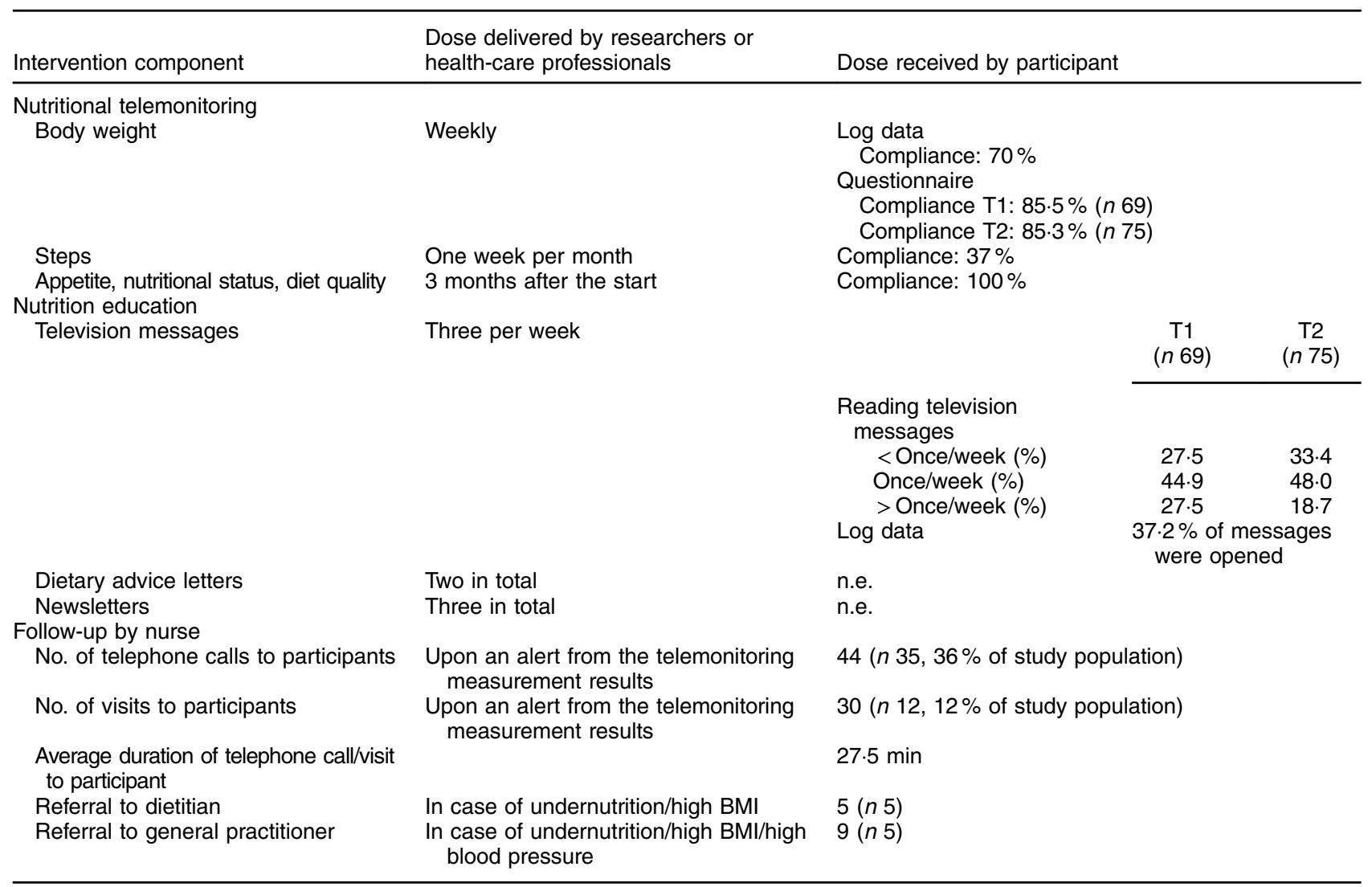

T1, 4.5 months after the start of the study; T2, 6 months after the start of the study; n.e., not evaluated.

†The PhysioDom Home Dietary Intake Monitoring (HDIM) intervention consisted of telemonitoring, nutrition education and follow-up by a nurse, implemented in a health-care setting among Dutch community-dwelling older adults from April 2016 to June 2017. 
television messages on a weekly basis. Log data revealed that $37.2 \%$ of the television messages were opened by participants. Regarding follow-up of telemonitoring measurements by a nurse, $36 \%$ of the participants received on average 1.2 phone calls and $12 \%$ were visited on average 2.8 times at home. These contact moments took on average $27.5 \mathrm{~min}$. Five participants were referred to a dietitian and another five were referred to their general practitioner.

\section{Acceptability}

Table 5 shows that participants were satisfied about the project with an average score of 4.0 out of 5 ; this score was higher at T1 and slightly lower at T2. Almost all acceptability scores decreased slightly from $\mathrm{T} 1$ to $\mathrm{T} 2$, although these decreases were not statistically significant. Of the four UTAUT constructs, effort expectancy and facilitating conditions were rated highest with scores between 3.8 and 4.0 at $\mathrm{T} 1$ and $\mathrm{T} 2$, indicating that participants found that the intervention technology was easy to use and that the helpdesk supported the use of the technology. Interviews with participants revealed that they were generally positive about the technology. The intended use of it was understood well. Nevertheless, many interviewees struggled with some intervention tools, mainly the weighing scale and the television channel. Remarkably, nurses were more negative about the participants' ease of use of the technology than participants themselves. Nurses were often asked to help with the telemonitoring measurements. Nurses mentioned that 'this generation' is not used to technology, that participants needed a lot of help, and that they easily became frustrated or stressed when technology was not working properly. With regards to facilitating conditions, interviewed participants perceived the helpdesk as friendly and helpful. However, participants also noticed that it was not always accessible, and some felt apprehension to approach the helpdesk: 'I don't want to be a nuisance to anyone'. Performance expectancy was rated 3.4 and 3.3 at $\mathrm{T} 1$ and $\mathrm{T} 2$, respectively, indicating that participants were neutral to positive about the contribution of the intervention to gains in a healthy diet, levels of physical activity, or health. All interviewees indicated that at least one intervention component gave new insight into their behaviour or health (e.g. the pedometer). However, the extent to which these insights impacted behaviour and health was highly variable among interviewees. Some interviewees indicated that the intervention helped to improve diet and physical activity and called the intervention 'stimulating', 'increasing awareness of own habits' and 'providing useful insights for improving one's diet'.

Table 5 Acceptability of the PhysioDom HDIM interventiont as rated by participants and health-care professionals

\begin{tabular}{|c|c|c|c|c|c|c|c|}
\hline & \multicolumn{3}{|l|}{$\mathrm{T} 1$} & \multicolumn{3}{|c|}{ T2 } & \multirow[b]{2}{*}{$\begin{array}{c}\text { Cronbach's } a, \\
\text { T1/T2 }\end{array}$} \\
\hline & $\begin{array}{c}\text { Mean } \\
\text { (range 1-5) }\end{array}$ & SD & $n$ & $\begin{array}{c}\text { Mean } \\
\text { (range 1-5) }\end{array}$ & SD & $n$ & \\
\hline \multicolumn{8}{|l|}{ Participants } \\
\hline \multicolumn{8}{|l|}{ General } \\
\hline I am satisfied about the project in general & $4 \cdot 1$ & $0 \cdot 8$ & 70 & 3.9 & 0.9 & 75 & \\
\hline I am satisfied about the nutrition part of the project & $4 \cdot 0$ & 0.8 & 70 & $3 \cdot 8$ & 0.8 & 75 & \\
\hline I am satisfied about the physical activity part of the project & $4 \cdot 1$ & 0.8 & 70 & 3.8 & 0.8 & 75 & \\
\hline I am satisfied about the contact with the nurse & $4 \cdot 3$ & 0.6 & 23 & 3.8 & 0.8 & 24 & \\
\hline Performance expectancy & 3.4 & 0.7 & 70 & 3.3 & 0.7 & 75 & $0.71 / 0.76$ \\
\hline Effort expectancy & 3.9 & 0.7 & 70 & $3 \cdot 8$ & 0.7 & 75 & $0.73 / 0.79$ \\
\hline Social influence & $3 \cdot 2$ & $1 \cdot 2$ & 69 & $3 \cdot 1$ & $1 \cdot 1$ & 73 & $0.94 / 0.89$ \\
\hline Facilitating conditions & 4.0 & 0.7 & 70 & 4.0 & 0.7 & 75 & $0.91 / 0.85$ \\
\hline \multirow[t]{2}{*}{ Behavioural intent } & - & - & - & $3 \cdot 3$ & 0.8 & 66 & \\
\hline & $\begin{array}{c}\text { Mean } \\
\text { (range 1-5) }\end{array}$ & SD & $n$ & & & & Cronbach's $a$ \\
\hline \multicolumn{8}{|l|}{ Health-care professionals } \\
\hline General & & & 8 & & & & \\
\hline I am satisfied about the project in general & 3.5 & 0.8 & & & & & \\
\hline Performance expectancy & & & 8 & & & & \\
\hline Gains for job performance & $2 \cdot 6$ & 0.6 & & & & & 0.71 \\
\hline Gains for client & & & & & & & -0.81 \\
\hline The project is useful to monitor nutritional status & $4 \cdot 1$ & 0.4 & & & & & \\
\hline The project is useful to coach clients concerning physical activity & 3.9 & 0.4 & & & & & \\
\hline The project is useful to coach clients concerning nutrition & 3.9 & 0.4 & & & & & \\
\hline The project can contribute to a better health for clients & $4 \cdot 1$ & 0.4 & & & & & \\
\hline Effort expectancy & $3 \cdot 2$ & 0.5 & 8 & & & & 0.80 \\
\hline Social influence & & & 8 & & & & 0.26 \\
\hline I felt supported by colleagues in implementing the intervention & $3 \cdot 3$ & 0.5 & & & & & \\
\hline The support of colleagues is important to me & 4.4 & 0.5 & & & & & \\
\hline Behavioural intent & $2 \cdot 3$ & 1.0 & 8 & & & & \\
\hline
\end{tabular}

T1, 4.5 months after the start of the study; T2, 6 months after the start of the study.

†The PhysioDom Home Dietary Intake Monitoring (HDIM) intervention consisted of telemonitoring, nutrition education and follow-up by a nurse, implemented in a health-care setting among Dutch community-dwelling older adults from April 2016 to June 2017. 
Other interviewees mentioned that the intervention had little to no effect on them or their health and called the telemonitoring results and advices 'unnecessary', 'not for me' or 'just for fun, nothing more'. They were already satisfied with their health, found that the supervision of a health-care professional was sufficient for them, or they already knew what they needed. While interviewees with a positive perspective appeared to be a majority, the distinction between the two points of view was not absolute. Most interviewees expressed both positive and negative sentiments regarding the performance of the intervention. Participants were neutral about social influence, with scores of 3.2 and 3.1 at $\mathrm{T} 1$ and $\mathrm{T} 2$, respectively. In the interviews, participants mentioned that they received positive feedback from their social environment about their participation, but that the social environment had little influence on their experience with the intervention. Participation was seen as a personal undertaking and the decision to participate was their own. Finally, participants were neutral about their intent to use the intervention more often. In the interviews, participants mentioned that it would be 'just more of the same', 'too time/energy consuming' or '[I] have already gotten everything out of this experience'. Only five interviewees preferred to continue participation, mentioning the stimulation to be physically active and the structure the intervention provided: 'Yes, the project stimulates. Now, I'll have to continue with it myself' and 'Like I said, it's about structure in your life [...]. This [intervention] is just a part of that'.

Acceptability scores of nurses were slightly lower than those of participants with an average satisfaction score of 3.5. Concerning performance expectancy, nurses were more positive about the gains of the intervention for their clients than the gains for their own job performance. Nurses called the project an addition to care, promising for the future, enabling ageing in place and possibly costsaving. However, the intervention was difficult to fit in the nurses' schedules as it took them a lot of time next to their normal working hours. Nurses made time for this when possible, but primary care needs of their clients had priority, sometimes resulting in postponing interventionrelated tasks. Effort expectancy was rated neutral with a 3.2. Nurses found the layout of the project website not clear and intuitive, and they could not report follow-up of telemonitoring measurements on it. As a result, they had to keep their own administration next to the website. Nurses also reported interferences of the website. Finally, nurses found it difficult to provide follow-up of telemonitoring measurements of participants who did not receive home care. Nurses did not know the background or medical history of these participants and found it therefore difficult to assess telemonitoring results properly. With regards to social influence, nurses were neutral to slightly positive about support of their colleagues, while they indicated that support of colleagues is important to them. The interviews revealed that cooperation with colleagues within the project team was good, but that support of other colleagues and the management of the health-care organisation was lacking. Cooperation with researchers was experienced as pleasant, although some nurses preferred to have more personal meetings instead of telephone meetings. Finally, nurses were negative about participation in a possible continuation of the intervention, with lack of time as the main reason. Some nurses only wanted to continue if the project website would be improved including notifications via email and integration of the website with the clients' electronic health records.

\section{Intervention's mechanisms of impact}

Table 6 shows determinants of intention to use the intervention. The first model with the participant characteristics of age, sex, education, cognitive functioning and physical functioning explained only $9 \%$ of the variation in intention to use PhysioDom HDIM. None of these characteristics was significantly associated with intention to use PhysioDom HDIM. The percentage of explained variance increased to $45 \%$ after adding the UTAUT constructs to the model. Performance expectancy and social influence significantly increased explained variance by 26 and $5 \%$, respectively. Effort expectancy increased explained variance by $4 \%(P=0.07)$. The final model showed that performance expectancy and social influence were significant predictors of intention to use PhysioDom HDIM.

Table 7 shows the influence of the process indicators acceptability and dose received on effects of the intervention on nutritional status and DHD-FFQ scores for fruits, vegetables, fibre, protein, and physical activity. No significant associations of acceptability and dose received with effects of the intervention were observed.

\section{Discussion}

The current process evaluation provided insight into how PhysioDom HDIM was implemented and received by participants and nurses. The intervention was largely implemented as intended with higher satisfaction rates among participants than among nurses. Both participants and nurses mentioned concerns regarding performance and effort expectancy of the intervention. Furthermore, participants' intention to use the intervention was predicted by performance expectancy and social influence. Acceptability and dose received were not associated with intervention effects.

Concerning the reach of this intervention, about $20 \%$ of the participants dropped out. Dropouts were older, less healthy and more care-dependent than completers. This is similar to the experience of another eHealth study among older adults in which dropouts were older and participants dropped out due to health deterioration ${ }^{(25)}$. In two other studies, having one or more chronic conditions was 
Table 6 Determinants of intention to use the PhysioDom HDIM intervention† ( $n$ 63)

\begin{tabular}{|c|c|c|c|c|c|c|c|c|c|c|}
\hline & \multicolumn{2}{|c|}{ Block 1} & \multicolumn{2}{|c|}{ Block 2} & \multicolumn{2}{|c|}{ Block 3} & \multicolumn{2}{|c|}{ Block 4} & \multicolumn{2}{|c|}{ Final model } \\
\hline $\begin{array}{l}R^{2} \\
F \text { change } \\
P \text { value }\end{array}$ & \multicolumn{2}{|c|}{$\begin{array}{l}0.09 \\
1.18 \\
0.33\end{array}$} & \multicolumn{2}{|c|}{$\begin{array}{r}0.35 \\
22.29 \\
0.00\end{array}$} & \multicolumn{2}{|c|}{$\begin{array}{l}0.39 \\
3.51 \\
0.07\end{array}$} & \multicolumn{2}{|c|}{$\begin{array}{l}0.44 \\
5.13 \\
0.03\end{array}$} & \multicolumn{2}{|c|}{$\begin{array}{l}0.45 \\
0.60 \\
0.44\end{array}$} \\
\hline & $\beta$ & $95 \% \mathrm{Cl}$ & $\beta$ & $95 \% \mathrm{Cl}$ & $\beta$ & $95 \% \mathrm{Cl}$ & $\beta$ & $95 \% \mathrm{Cl}$ & $\beta$ & $95 \% \mathrm{Cl}$ \\
\hline $\begin{array}{l}\text { Age } \\
\text { Sex } \\
\text { Education } \\
\text { Cognitive functioning } \neq \\
\text { Physical functioning§ } \\
\text { Performance expectancy } \\
\text { Effort expectancy } \\
\text { Social influence } \\
\text { Facilitating conditions }\end{array}$ & $\begin{array}{r}-0.16 \\
0.02 \\
-0.22 \\
0.20 \\
0.02\end{array}$ & $\begin{array}{l}-0.63,0.30 \\
-0.01,0.05 \\
-0.53,0.09 \\
-0.03,0.43 \\
-0.07,0.11\end{array}$ & $\begin{array}{r}-0.30 \\
0.01 \\
-0.09 \\
0.15 \\
-0.01 \\
0.59\end{array}$ & 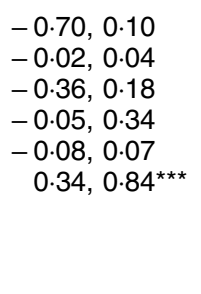 & $\begin{array}{r}-0.27 \\
0.00 \\
-0.09 \\
0.11 \\
-0.04 \\
0.50 \\
0.30\end{array}$ & $\begin{array}{c}-0.66,0.13 \\
-0.03,0.03 \\
-0.35,0.18 \\
-0.08,0.31 \\
-0.12,0.04 \\
0.23,0.76^{\star \star *} \\
-0.02,0.61\end{array}$ & $\begin{array}{r}-0.13 \\
0.01 \\
-0.03 \\
0.10 \\
-0.03 \\
0.40 \\
0.34 \\
0.19\end{array}$ & $\begin{array}{c}-0.53,0.27 \\
-0.02,0.04 \\
-0.29,0.23 \\
-0.08,0.29 \\
-0.11,0.05 \\
0.13,0.67^{\star \star} \\
0.03,0.65^{\star} \\
0.02,0.35^{\star}\end{array}$ & $\begin{array}{r}-0.11 \\
0.01 \\
-0.03 \\
0.10 \\
-0.03 \\
0.40 \\
0.29 \\
0.17 \\
0.11\end{array}$ & $\begin{array}{c}-0.52,0.29 \\
-0.02,0.04 \\
-0.29,0.23 \\
-0.09,0.29 \\
-0.10,0.05 \\
0.13,0.67^{\star *} \\
-0.05,0.62 \\
0.00,0.34^{\star} \\
-0.18,0.40\end{array}$ \\
\hline
\end{tabular}

Dependent variable: intention to use PhysioDom HDIM more often (range 1-5).

${ }^{\star} P<0.05,{ }^{\star \star} P<0.01,{ }^{\star \star \star} P<0.001$.

†The PhysioDom Home Dietary Intake Monitoring (HDIM) intervention consisted of telemonitoring, nutrition education and follow-up by a nurse, implemented in a health-care setting among Dutch community-dwelling older adults from April 2016 to June 2017.

$\ddagger$ Measured by Mini Mental State Examination.

§Measured by Short Physical Performance Battery.

Table 7 Influence of acceptability and dose received on effects of the PhysioDom HDIM intervention† on nutritional status and diet quality

\begin{tabular}{|c|c|c|c|c|c|c|c|c|c|c|c|c|c|c|}
\hline & \multicolumn{2}{|c|}{$\begin{array}{l}\text { MNA score, } \\
\triangle \mathrm{T} 1-\mathrm{T0}\end{array}$} & \multicolumn{2}{|c|}{$\begin{array}{l}\text { MNA score, } \\
\triangle \mathrm{T} 2-\mathrm{T0}\end{array}$} & \multicolumn{2}{|c|}{$\begin{array}{c}\text { DHD-FFQ } \\
\text { vegetables, } \\
\Delta \mathrm{T} 2-\mathrm{T0}\end{array}$} & \multicolumn{2}{|c|}{$\begin{array}{l}\text { DHD-FFQ } \\
\text { fruits, } \\
\Delta \mathrm{T} 2-\mathrm{T0}\end{array}$} & \multicolumn{2}{|c|}{$\begin{array}{l}\text { DHD-FFQ } \\
\text { fibre, } \\
\Delta \mathrm{T} 2-\mathrm{TO} 0\end{array}$} & \multicolumn{2}{|c|}{$\begin{array}{l}\text { DHD-FFQ } \\
\text { protein, } \\
\Delta \mathrm{T} 2-\mathrm{TO}\end{array}$} & \multicolumn{2}{|c|}{$\begin{array}{c}\text { DHD-FFQ } \\
\text { physical activity, } \\
\Delta \mathrm{T} 2-\mathrm{T0}\end{array}$} \\
\hline & $\beta$ & $95 \% \mathrm{Cl}$ & $\beta$ & $95 \% \mathrm{Cl}$ & $\beta$ & $95 \% \mathrm{Cl}$ & $\beta$ & $95 \% \mathrm{Cl}$ & $\beta$ & $95 \% \mathrm{Cl}$ & $\beta$ & $95 \% \mathrm{Cl}$ & $\beta$ & $95 \% \mathrm{Cl}$ \\
\hline \multicolumn{15}{|l|}{ Acceptability } \\
\hline Performance expectancy & 0.21 & $-0.65,1.06$ & 0.41 & $-0.58,1.39$ & -0.03 & $-1.27,1.22$ & 0.42 & $-0.45,1.29$ & 0.17 & $-0.45,0.78$ & 0.17 & $-1.86,1.32$ & -0.70 & $-2.39,0.98$ \\
\hline Effort expectancy & 0.80 & $-0.10,1.69$ & 0.07 & $-0.96,1.10$ & -0.06 & $-1.37,1.25$ & -0.57 & $-1.49,0.34$ & 0.39 & $-0.26,1.03$ & 0.17 & $-1.50,1.84$ & -0.21 & $-1.98,1.57$ \\
\hline Social influence & 0.35 & $-0.27,0.97$ & 0.20 & $-0.51,0.91$ & -0.28 & $-1.19,0.64$ & -0.43 & $-1.06,0.21$ & 0.13 & $-0.32,0.58$ & -0.84 & $-2.01,0.32$ & -0.14 & $-1.38,1 \cdot 10$ \\
\hline Facilitating conditions & -0.87 & $-1.87,0.13$ & -0.34 & $-1.48,0.81$ & 0.03 & $-1.45,1.51$ & 0.72 & $-0.31,1.75$ & -0.57 & $-1.30,0.16$ & 0.87 & $-1.02,2.75$ & -1.08 & $-3.08,0.93$ \\
\hline \multicolumn{15}{|l|}{ Dose received } \\
\hline $\begin{array}{l}\text { Adherence body weight } \\
\text { measurements§ }\end{array}$ & 0.49 & $-0.92,1.91$ & 0.58 & $-1 \cdot 07,2 \cdot 23$ & 0.54 & $-1 \cdot 18,2 \cdot 27$ & -0.42 & $-1 \cdot 80,0.96$ & 0.15 & $-0.71,1.01$ & 1.38 & $-0.76,3.52$ & $-1 \cdot 54$ & $-3 \cdot 80,0.73$ \\
\hline Percentage of messages opened $\neq$ & 0.01 & $-1.93,1.95$ & 0.03 & $-2 \cdot 13,2 \cdot 19$ & 0.99 & $-1.34,3.32$ & 0.64 & $-1 \cdot 24,2.51$ & 0.48 & $-0.68,1.63$ & 0.56 & $-2.37,3.49$ & -0.82 & $-3.91,2 \cdot 28$ \\
\hline $\begin{array}{l}\text { Frequency of contact with nurse from } \\
\text { project }\end{array}$ & -0.02 & $-0.30,0.25$ & 0.00 & $-0.31,0.32$ & -0.17 & $-0.50,0.17$ & 0.10 & $-0.17,0.37$ & -0.00 & $-0.17,0.16$ & -0.15 & $-0.58,0.28$ & -0.08 & $-0.53,0.36$ \\
\hline
\end{tabular}

MNA, Mini Nutritional Assessment; DHD-FFQ, Dutch Healthy Diet Food Frequency Questionnaire; T0, baseline; T1, 4.5 months after the start of the study; T2, 6 months after the start of the study.

The PhysioDom Home Dietary Intake Monitoring (HDIM) intervention consisted of telemonitoring, nutrition education and follow-up by a nurse, implemented in a health-care setting among Dutch community-dwelling older adults from April 2016 to June 2017.

fConstructs from the Unified Theory of Acceptance and Use of Technology are combined in one model and adjusted for age, sex, help from relatives, living situation and number of diagnoses.

$\S$ Adjusted for age and education. 
associated with lower adherence to an eHealth intervention $^{(26,27)}$. This has implications for the expectation that eHealth improves health-care access and health equity, as the present study and other studies show that older age and poorer health are related to higher dropout or lower adherence. Therefore, health disparities may still remain for persons who are less able or willing to keep up with eHealth $^{(28)}$. For future generations of older adults with higher computer literacy this issue might be less problematic. Nevertheless, research should focus on how the reach towards these groups can be improved or by considering other modalities than eHealth to promote health in non-adopters of eHealth ${ }^{(29)}$.

In general, participants were satisfied about the intervention. When looking at the UTAUT constructs, we found that participants rated effort expectancy and facilitating conditions the highest. Participants were more neutral about performance expectancy and social influence. The results for effort expectancy and social influence seem contradictory to the nurses' views. The nurses were more negative about the participants' ease of use of the intervention than participants themselves. Furthermore, nurses supported many participants in performing the telemonitoring measurements, but participants were neutral about the role of social influence. This could be partly due to the operationalisation of social influence, as participants might not see their nurses as 'important others' but rather think of relatives. Another explanation might be that the nurses especially observed participants who frequently needed help. All in all, taking account of the views of both participants and nurses is relevant as both have an essential role in successful implementation.

However, social influence was a predictor for intention to use the intervention, together with performance expectancy. Performance expectancy has been identified as an important predictor of use ${ }^{(10-12,30,31)}$. The literature is divided about the role of social influence in eHealth adoption. According UTAUT, social influence is a significant predictor in mandatory settings only and studies did not find an association between social influence and intention to use eHealth among older adults ${ }^{(11,12,14)}$. However, our study and others did find an association $^{(10,32-35)}$. This might be explained by the way social influence is operationalised in studies, or by the suggestion that the role of social influence is dependent on the context $^{(12,14)}$. Many technology acceptance models have reduced social influence to the construct of subjective norm (i.e. perception that important others think he/she should or should not use technology), but social influence also encompasses the influence of technology suppliers, health-care professionals and the help of relatives ${ }^{(10,36)}$. Models concerning technology acceptance by older adults should pay attention to this more complex role of social influence.

Unexpectedly, no associations of the process indicators dose received and acceptability with effects of the intervention were found. Previous research suggests that intervention adherence is related to better outcomes ${ }^{(37-40)}$. However, other studies did not find such an association or presented mixed results ${ }^{(27,41-43)}$. It could be that the process indicators in the present study and the way they were measured did not capture implementation sufficiently. For example, self-report could have introduced recall bias. Another explanation might be that the relationship between process indicators and outcomes is more complex, for example not following a linear relationship or influenced by sociodemographic factors or personality traits $^{(41,42)}$. Future studies should continue to include process measures to unravel interventions' mechanisms of impact and to unveil successful intervention elements.

The current study made use of evaluation frameworks to underpin the evaluation strategy. The Medical Research Council and UTAUT frameworks have been widely used for process evaluation and technology acceptance, respectively. One of the objections concerning UTAUT, however, is that this model lacks important determinants of technology use that are specific to community-dwelling older adults, such as cognitive and physical functioning and several contextual factors ${ }^{(10)}$. This was taken into account by including cognitive and physical functioning in the analyses, together with the UTAUT constructs. Furthermore, both quantitative and qualitative data were used to capture an in-depth overview of how the intervention was implemented and received. Collecting data from both participants and nurses resulted in two complementary perspectives on the intervention. A limitation of the study might be recall bias among nurses concerning their implementation of the intervention. Nurses were asked about their frequency and duration of intervention activities half-way during the intervention and were reminded at the end of the intervention to record the contact moments with participants. This might have obscured the association between intervention dose and effects.

Based on the present study, some implications for future research and practice are presented. First, nurses found it difficult to perform follow-up of telemonitoring results of participants who did not receive home care. This suggests that telemonitoring can better be implemented within a care context in which nurses know the telemonitoring recipients. Second, telemonitoring has the purpose to partly replace care from health-care professionals. However, some of our participants needed much guidance from nurses in performing telemonitoring measurements, suggesting that the current intervention, implemented among the current generation, requires more guidance from nurses than desired. Improved usability may reduce the need for guidance, as well as the expectation that future generations have better computer literacy. Third, the study underlines the importance of user-centred design in developing eHealth intervention for older adults. This process evaluation revealed several aspects that would hinder long-term use of the intervention, such 
as the usability and interoperability of the nurses' website, the perceived need for the intervention, and the usability and attractiveness of the television channel. Although we have pre-tested the telemonitoring technology in a pilot study, it is recommended that end users and other relevant stakeholders are even more involved in iterative development cycles of eHealth applications ${ }^{(44,45)}$.

\section{Conclusion}

To conclude, the PhysioDom HDIM intervention was feasible to implement with good satisfaction among participants, but lower satisfaction among nurses. Nutritional telemonitoring interventions should be user-friendly so that telemonitoring measurements can be performed without guidance from nurses; and should fit with working procedures from nurses for successful adoption and implementation. The perceived benefits of the intervention and social influence predicted the participants' intention to use the intervention, which can be used as strategies for future intervention design and implementation. Future research should focus on how to enlarge the reach of eHealth interventions to more frail older adults and on unravelling mechanisms of impact.

\section{Acknowledgements}

Acknowledgements: The authors would like to thank all participants, health-care professionals and the boards of Zorggroep Noordwest-Veluwe and Opella. Furthermore, they would like to thank Iris Geigenmüller and Joline Wierda for their contribution to the data collection and data analysis of this study. Financial support: This work was supported by the European Union (grant number CIPICT-PSP-2013-7). The European Union had no role in the design, analysis or writing of this article. Conflict of interest: None. Authorship: M.N.v.D.-v.A. coordinated implementation of the study, analysed the data and drafted the article. S.S. and A.C.R. contributed to the study design. L.C.P.G.M.d.G., J.H.M.d.V., A.H.-N., S.S. and A.C.R. critically read and revised the manuscript. Ethics of buman subject participation: This study was conducted according to the guidelines laid down in the Declaration of Helsinki and all procedures involving human subjects were approved by the ethics committee of Wageningen University. Written informed consent was obtained from all subjects.

\section{References}

1. Norman K, Pichard C, Lochs H et al. (2008) Prognostic impact of disease-related malnutrition. Clin Nutr 27, 5-15.

2. Kaiser MJ, Bauer JM, Ramsch C et al. (2010) Frequency of malnutrition in older adults: a multinational perspective using the mini nutritional assessment. J Am Geriatr Soc $\mathbf{5 8}$, $1734-1738$.
3. Schilp J, Kruizenga HM, Wijnhoven HA et al. (2012) High prevalence of undernutrition in Dutch community-dwelling older individuals. Nutrition 28, 1151-1156.

4. Elia M, Zellipour L \& Stratton RJ (2005) To screen or not to screen for adult malnutrition? Clin Nutr 24, 867-884.

5. Van Doorn-van Atten MN, Haveman-Nies A, van Bakel M et al. (2018) Effects of a multi-component nutritional telemonitoring intervention on nutritional status, diet quality, physical functioning, and quality of life of communitydwelling older adults. Br J Nutr 119, 1185-1194.

6. Saunders RP, Evans MH \& Joshi P (2005) Developing a process-evaluation plan for assessing health promotion program implementation: a how-to guide. Health Promot Pract 6, 134-147.

7. Moore GF, Audrey S, Barker M et al. (2015) Process evaluation of complex interventions: Medical Research Council guidance. BMJ 350, h1258.

8. World Health Organization (2016) Global Diffusion of eHealth: Making Universal Health Coverage Achievable. Report of the Third Global Survey on eHealth. Geneva: WHO.

9. Peek ST, Wouters EJ, Luijkx KG et al. (2016) What it takes to successfully implement technology for aging in place: focus groups with stakeholders. J Med Internet Res 18, e98.

10. Peek ST, Wouters EJ, van Hoof J et al. (2014) Factors influencing acceptance of technology for aging in place: a systematic review. Int J Med Inform 83, 235-248.

11. Venkatesh V, Morris MG, Davis GB et al. (2003) User acceptance of information technology: toward a unified view. MIS Q 27, 425-478.

12. de Veer AJE, Peeters JM, Brabers AEM et al. (2015) Determinants of the intention to use e-Health by community dwelling older people. BMC Health Serv Res 15, 103.

13. Kohnke A, Cole M \& Bush R (2014) Incorporating UTAUT predictors for understanding home care patients' and clinician's acceptance of healthcare telemedicine equipment. $J$ Technol Manag Innovat 9, 29-41.

14. Cimperman M, Makovec Brencic M \& Trkman P (2016) Analyzing older users' home telehealth services acceptance behaviour - applying an Extended UTAUT model. Int J Med Inform 90, 22-31.

15. van Doorn-van Atten MN, Haveman-Nies A, Pilichowski P et al. (2018) Telemonitoring to improve nutritional status in community-dwelling elderly: design and methods for process and effect evaluation. BMC Geriatr (In the Press).

16. Kaiser MJ, Bauer JM, Ramsch C et al. (2009) Validation of the Mini Nutritional Assessment Short-Form (MNA-SF): a practical tool for identification of nutritional status. $J$ Nutr Health Aging 13, 782-788.

17. Wilson MM, Thomas DR, Rubenstein LZ et al. (2005) Appetite assessment: simple appetite questionnaire predicts weight loss in community-dwelling adults and nursing home residents. Am J Clin Nutr 82, 1074-1081.

18. van Lee L, Feskens EJ, Meijboom S et al. (2016) Evaluation of a screener to assess diet quality in the Netherlands. Br J Nutr 115, 517-526.

19. Voedingscentrum (2017) Ondervoeding. Eten bij ondervoeding en bij herstel na ziekte, vol. D752-20. https:// webshop.voedingscentrum.nl/pdf/D752-20.pdf (accessed August 2018).

20. Folstein MF, Folstein SE \& McHugh PR (1975) 'Mini-mental state'. A practical method for grading the cognitive state of patients for the clinician. J Psychiatr Res 12, 189-198.

21. Vellas B, Villars H, Abellan G et al. (2006) Overview of the MNA - its history and challenges. J Nutr Health Aging 10, 456-463.

22. Guralnik JM, Simonsick EM, Ferrucci L et al. (1994) A short physical performance battery assessing lower extremity function: association with self-reported disability and prediction of mortality and nursing home admission. J Gerontol 49, M85-M94. 
23. Laan W, Zuithoff NP, Drubbel I et al. (2014) Validity and reliability of the Katz-15 scale to measure unfavorable health outcomes in community-dwelling older people. $J$ Nutr Health Aging 18, 848-854.

24. Gezondheidsraad (2006) Richtlijnen Goede Voeding 2006. Den Haag: Gezondheidsraad.

25. Hirani SP, Beynon M, Cartwright M et al. (2014) The effect of telecare on the quality of life and psychological wellbeing of elderly recipients of social care over a 12-month period: the Whole Systems Demonstrator cluster randomised trial. Age Ageing 43, 334-341.

26. Guzman-Clark JR, van Servellen G, Chang B et al. (2013) Predictors and outcomes of early adherence to the use of a home telehealth device by older veterans with heart failure. Telemed J E Health 19, 217-223.

27. Kelders MS, Van Gemert-Pijnen EWCJ, Werkman A et al. (2011) Effectiveness of a web-based intervention aimed at healthy dietary and physical activity behavior: a randomized controlled trial about users and usage. J Med Internet Res 13, e32.

28. Eysenbach G (2001) What is e-health? J Med Internet Res $\mathbf{3}$, E20.

29. Zbib A, Hodgson C \& Calderwood S (2011) Can eHealth tools enable health organizations to reach their target audience? Healthc Manage Forum 24, 155-159.

30. Lee C \& Coughlin J (2014) PERSPECTIVE: Older adults' adoption of technology: an integrated approach to identifying determinants and barriers. J Prod Innovat Manag 32, 747-759.

31. Peeters JM, de Veer AJ, van der Hoek L et al. (2012) Factors influencing the adoption of home telecare by elderly or chronically ill people: a national survey. J Clin Nurs 21, 3183-3193.

32. Hsu YC, Tsai CH, Kuo YM et al. (2016) Telecare services for elderly: predictive factors of continued use intention. Open Biomed Eng J 10, 82-90.

33. Kijsanayotin B, Pannarunothai S \& Speedie SM (2009) Factors influencing health information technology adoption in Thailand's community health centers: applying the UTAUT model. Int J Med Inform 78, 404-416.

34. Or CKL, Karsh B-T, Severtson DJ et al. (2011) Factors affecting home care patients' acceptance of a web-based interactive self-management technology. J Am Med Inform Assoc 18, 51-59.

35. Chen K \& Chan AH-S (2013) Use or non-use of gerontechnology - a qualitative study. Int J Environ Res Public Health 10, 4645-4666.

36. Gramstad A, Storli SL \& Hamran T (2014) Older individuals' experiences during the assistive technology device service delivery process. Scand J Occup Ther 21, 305-312.

37. Couper MP, Alexander GL, Zhang N et al. (2010) Engagement and retention: measuring breadth and depth of participant use of an online intervention. J Med Internet Res $\mathbf{1 2}$, e 52 .

38. Norman GJ, Zabinski MF, Adams MA et al. (2007) A review of eHealth interventions for physical activity and dietary behavior change. Am J Prev Med 33, 336-345.e316.

39. Mattila E, Orsama AL, Ahtinen A et al. (2013) Personal health technologies in employee health promotion: usage activity, usefulness, and health-related outcomes in a 1-year randomized controlled trial. JMIR Mhealth Uhealth 1, e16.

40. Neve M, Morgan PJ, Jones PR et al. (2010) Effectiveness of web-based interventions in achieving weight loss and weight loss maintenance in overweight and obese adults: a systematic review with meta-analysis. Obes Rev 11, 306-321.

41. Wildeboer G, Kelders SM \& van Gemert-Pijnen JEWC (2016) The relationship between persuasive technology principles, adherence and effect of web-based interventions for mental health: a meta-analysis. Int J Med Inform 96, 71-85.

42. Donkin L, Hickie BI, Christensen H et al. (2013) Rethinking the dose-response relationship between usage and outcome in an online intervention for depression: randomized controlled trial. J Med Internet Res 15, e231.

43. Dane AV \& Schneider BH (1998) Program integrity in primary and early secondary prevention: are implementation effects out of control? Clin Psychol Rev 18, 23-45.

44. Matthew-Maich N, Harris L, Ploeg J et al. (2016) Designing, implementing, and evaluating mobile health technologies for managing chronic conditions in older adults: a scoping review. JMIR Mhealth Uhealth 4, e29.

45. Gustafson DH Jr, Maus A, Judkins J et al. (2016) Using the NIATx model to implement user-centered design of technology for older adults. JMIR Hum Factors 3, e2. 